\title{
Application of Random Forest to the Prototying of the 3D Garment
}

\author{
Shuaijun Wang, Ye Yuan* and Hongli Dun \\ School of Information Engineering, Beijing Institute of Fashion Technology, Beijing 100029, China \\ ${ }^{*}$ Corresponding author
}

\begin{abstract}
A digital 3D scanning technique for human body is proposed. Firstly, feature point recognition is carried out on point cloud model of 3D sweeping human body. This method adopts the more mature random forest algorithm, and the algorithm has higher recognition effect. Then, based on the recognition of the feature points, the clothing garment of $3 D$ clothing was constructed by Bezier curve, including the shoulder piece, the chest piece, the abdominal piece and the back piece, and the clothing was simulated. Then cut parts of the garment using the level of B-spline editing, making it more suitable for 3D human body model. Finally, the Euler algorithm is used to simulate the 3D garment. The experimental results show that the method is simple, effective and feasible.
\end{abstract}

Keywords-3D scanning body; random forest prototying of the 3D Garment

\section{INTRODUCTION}

To achieve from the simple bulk production of clothing to personalized production of clothing industry transformation and upgrading, so that the production of clothing with cultural creativity and service content. 3D human body .

modeling has become the focus of the apparel industry research area, to develop a simple high-quality 3D human model imminent, on the basis of this new feature recognition, curve construction, surface structure algorithm to study the new 3D clothing modeling, in order to achieve Virtual clothing, two-dimensional clothing piece suture, which will become the garment enterprises to adapt to changes in the market, greatly enhance the economic efficiency and effective means of enterprise competition.

The application of digital technology ${ }^{[1-4]}$ in clothing mainly refers to the realization of 3D human body measurement, 3D garment design, 3D garment suture and 3D modeling, 3D fitting system and 3D garment display on computer. The ultimate goal is to represent the final design results on a virtual reality model without physical clothing, and to achieve significant savings in time and money by creating an interactive platform. The whole point is that it will not only improve Clothing production efficiency, more importantly, to improve the quality of clothing design. Therefore, as the clothing of the three-dimensional technology of digital garments has become a worldwide problem, all countries in the positive of this technology in research and development.

\section{FEATURE RECOGNITION OF 3D HUMAN}

In order to improve the accuracy of $3 \mathrm{D}$ human feature recognition and overcome the limitations of traditional methods, a 3D human feature recognition algorithm based on stochastic forest is proposed. Based on the segmentation of point cloud model, random forest algorithm is used to find the global maximum The splitting criterion of the superior feature was used to statistically identify the key parts of 3D human neck, shoulder, armpit, chest, waist and buttocks. The algorithm proposed in this paper does not use triangular meshes to reconstruct the model, but directly uses the point cloud image obtained by $3 \mathrm{D}$ human body to represent the human body surface. This method reduces the time complexity and the storage complexity.

The stochastic forest algorithm ${ }^{[5-8]}$ uses the bagging model to construct a number of independent trees by using a stochastic, random set of training sample sets and stochastic feature sets. The algorithm uses several independent decision tree to vote for the prediction As a result, the algorithm flow is as follows:

Step1.Useing Bagging ${ }^{[9-12]}$ to form the different training set. Suppose the number of samples in the original training set is $\mathrm{N}$, and randomly select $\mathrm{M}$ samples from the original training set to form a new training set to generate a classification tree.

Step2.The above steps are repeated until a number of classification trees are generated.

Step3.For each leaf node, randomly select a variable based on this point. According to this variable $v$, the optimal partitioning method is calculated. The splitting of the leaf node is calculated according to the gain and the gain of the node as follows:

$$
L\left(v_{j}\right)=\sum_{i=1}^{N} p_{i}^{j}\left(1-p_{i}^{j}\right)
$$

$j$ represents the $j$-th tree, and $i$ represents the $i$-th classification result, $\mathrm{N}$ represents the total classification tree.The probability of a leaf node on each tree is expressed as: $p_{j}=\left\{p_{1}^{j}, \ldots, p_{K}^{j}\right\} \cdot p_{j}$ are classified at leaf nodes, then the left and right leaf nodes probability:

$$
p_{j l s}=\left\{p_{1}^{j l s}, \ldots, p_{K}^{j l s}\right\} \quad p_{j \mathrm{rs}}=\left\{p_{1}^{j r s}, \ldots, p_{K}^{j r s}\right\}
$$

$l$ is the left, $S$ is the set of samples, $p_{1}^{j l s}$ is the splitting left node of set $S$ at $p_{j}$ leaf node, $p_{1}^{j r s}$ is the splitting right node of set $S$ at $p_{j}$ leaf node, and the gain of leaf node is: 


$$
\Delta L\left(v_{j} \diamond s\right)=L\left(v_{j}\right)-\frac{\left|v_{j l s}\right|}{\left|v_{j}\right|} L\left(v_{j l s}\right)-\frac{\left|v_{j r s}\right|}{\left|v_{j}\right|} L\left(v_{j r s}\right)
$$

At this point, the decision tree construction is complete.

Step4.Random selection of features on the classification of internal nodes of the regression tree to split: assuming a total of $x$ features, specify a positive integer $\mathrm{I}<<\mathrm{x}$; At each internal node,the Ith characteristic points are randomly extracted from the xth features as candidate characteristic points, and the best splitting method is selected to divide the nodes. The value of I remains constant throughout the growth of the forest.

Step5.Each tree grows freely without pruning.

Step6.Decision tree voting.A random forest is a collection of tree classifiers of $\left\{c\left(x, \theta_{k}\right), k=1, \ldots\right\}$,The meta-classifier $c\left(x, \theta_{k}\right)$ is a categorical regression tree constructed; $\mathrm{X}$ is the input vector, $\theta_{k}$ is an independent and identically distributed random vector, the random vector $\theta_{k}$ determines the growth process of a single tree; and the random forest constructs a different training set to increase the difference between the classification models, thus improving the extrapolation prediction ability of the combined classification model. Through $\mathrm{k}$ rounds training to obtain a classification model sequence $\left\{c_{1}(x), c_{2}(x), \ldots, c_{k}(x)\right\}$, and then use them to form a multi-classification model system, and use the simple majority voting method to get the final final classification decision of random forest:

$$
C X=\arg \max _{Y} \sum_{i=1}^{k} I\left(c_{i}(x)=Y\right)
$$

The algorithm of feature points recognition based on stochastic forest algorithm in 3D scanning human point cloud model is as follows:

Step1.The 3D scanned human body was pretreated by Geomagic Stadio 11 software to remove the unnecessary noise points in the point cloud model which may affect the feature recognition.

Step2.The 3D scanning human body is divided into 6 areas to be recognized, which are neck, shoulder, armpit, chest, waist and hips, according to the area of the 3D feature.

Step3. Using Bagging method to form a different training set for each region, using a randomly generated sample set to generate a number of independent trees, to build a random forest. According to the proportion of the human body (assuming the body height of 1 ), the regional feature points from the region within the total number of candidate points to determine the percentage points.

Step4.The probability of the leaf node to be recognized as a leaf node and the node gain value are respectively calculated according to the definition formula (1), (2), (3).

Step5.If the value of the leaf node is larger than the standard value, it is determined that the point is the candidate feature point, otherwise the point is not the candidate feature point.

Step6. A voting is performed for each candidate feature point, and the voting proportion of each candidate feature point is calculated according to the definition formula (4). And the candidate feature points with the ratio of more than $90 \%$ are used as final feature points.

Step7.The feature points obtained by fitting the abovementioned feature points by the cubic B-spline are obtained, and the characteristic curves of the characteristic regions are obtained. The length of each characteristic curve is calculated and the curve length is taken as the corresponding human body circumference size value.

In order to verify the feasibility of the algorithm, the simulation experiment using $\mathrm{C}++$ development environment and OpenGL platform for the application of this algorithm for 3D models were identified. Firstly, the human body model of three-dimensional scanning is selected to read into the $\mathrm{C}++$ development environment. Then, the model is simplified and the point cloud model of the human body model is obtained on the basis of simplification. Then, the simplified point cloud model Divided into: neck, shoulder, chest, waist, buttocks. Each part set reasonable threshold of feature recognition, in the threshold range of the human body model to identify the various parts of the feature points, and finally, the calculation of the location of the feature points and B-spline fitted data after the fit. The simulation results are shown in Figure I.

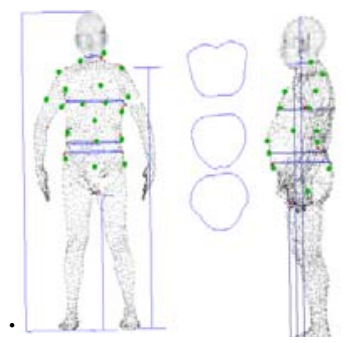

FIGURE I. FEATURE RECOGNITION RESULT

\section{RESEARCH ON SURFACE Modeling TECHNOLOGY}

Body features are geometric-oriented human model information with three types of attributes. Data containing the characteristics of the human body, a method for definition, for describing the positional relationship between the shape features. 3D human body model of the characteristic curve is the main feature, is the three-dimensional cut clothing designers are most concerned about. According to the feature point of the garment, the corresponding characteristic curve can be generated. The curve is generated by the relevant feature points (points defined according to the physical characteristics of the garment body) and the model sample points (points defined by the human body surface modeling). The characteristic curve reflects the feature size and the most important geometric features of the garment human body model. However, only the characteristic curve is not enough to express all the geometric shapes of the human body model, and the auxiliary modeling lines are needed to construct the characteristic surface. In this paper, the Bezier curve is used to 
construct the expandable garment surface. The algorithm has the advantages of high precision and low cost.

Garment fabrics have zero-Gaussian curvature properties, so a 3D garment prototype is constructed according to the class development surface rules of the attribute. The basic form of a ruled surface in a three-dimensional space is given by the Bézier surface ${ }^{[10]}$ :

$$
\begin{aligned}
& S(u, v)=\sum_{i=0}^{M} \sum_{j=0}^{1} P_{i j} B_{I}^{M}(u) B_{J}^{1}(V) \\
& =(1-v) \sum_{i=0}^{M} P_{i, 0} B_{i}^{M}(u)+v \sum_{i=0}^{M} P_{i, 1} B_{i}^{M}(u) m \\
& (u, v) \in[0,1] \times[0,1]
\end{aligned}
$$

amongthem $\quad P_{i, j} \in \mathrm{R}^{3}(i=0,1, \cdots, M, j=0,1) \quad, \quad B_{i}^{M}(u) \quad$ is $\quad$ a Bernstein function with order $\mathrm{M},{ }^{B_{J}^{1}(V)}$ is the Bernstein function of 1 , respectively. $\partial(u)$ and $\beta(u)$ represent as in (6) and (7), respectively.

$$
\begin{array}{r}
\partial(u)=\sum_{i=0}^{M} P_{i, 0} B_{i}^{M}(u) \\
\beta(u)=\sum_{i=0}^{M} P_{i, 1} B_{i}^{M}(u)
\end{array}
$$

The expression (5) is simplified to be: $S(u, v)=(1-v) \partial(\mathrm{u})+\mathrm{v} \beta(\mathrm{u})$, According to (5) and (6), $\partial(u)$ and $\beta(u)$ are Bezier curves determined by their respective control points $P_{0,0}, P_{1,0}, \cdots, P_{M, 0} \quad$ and $\quad P_{0,1}, P_{1,1} \cdots, P_{M, 1}$.Let $P_{0}^{0}(u)=P_{0,0}$, $P_{1}^{0}(u)=P_{1,0}, \ldots, P_{\mathrm{M}}^{0}(u)=P_{\mathrm{M}, 0}$ and $Q_{0}^{0}(u)=P_{0,1}, Q_{1}^{0}(u)=P_{1,1}, \ldots, \quad Q_{\mathrm{M}}^{0}(u)=P_{M, 1}$. Based on the de Casteljau algorithm, the recursive computation process is described below:

$$
\begin{array}{lll}
P_{0}^{0}(u) & & \\
P_{0}^{0}(u) & P_{0}^{1}(u) & \\
& & \\
P_{2}^{0}(u) & P_{1}^{1}(u) & \\
\vdots & \vdots & P_{0}^{\mathrm{M}-1}(u) \\
P_{M-1}^{0}(u) & P_{0}^{\mathrm{M}}(u) \\
& P_{M-1}^{1}(u) & P_{1}^{\mathrm{M}-1}(u) \\
P_{M}^{0}(u) & &
\end{array}
$$

Among them

$$
P_{i}^{k}(u)=(1-u) P_{i}^{k-1}(u)+u P_{i+1}^{k-1}(u), k=1,2, \ldots, M, i=0,1, \ldots, M-k
$$

Thus, the boundary curve $\partial(u)$ of $S(u, v)$ is given by:

$$
\partial(u)=\sum_{i=0}^{M-1} P_{i}^{1}(u) B_{i}^{M-1}(u)=\cdots=\sum_{i=0}^{M-k} P_{i}^{k}(u) B_{i}^{M-k}(u)=P_{M}^{0}(u)
$$

Similarly, $\beta(u)$ is recursively generated by the de Casteljau algorithm same as the $\mathrm{P}$ :

Since point $S\left(u_{0}, 0\right),\left(0 \leq u_{0} \leq 1\right)$ is on the boundary curve $\partial(u)$ of $S\left(u_{0}, 0\right)$, point $S\left(u_{0}, 1\right),\left(0 \leq u_{0} \leq 1\right)$ is on the boundary curve $\beta(u)$ of $S(u, v),\left(0 \leq u_{0} \leq 1\right)$, The straight line $S\left(u_{0}, 0\right)\left(S\left(u_{0}, 1\right)\right.$ constitutes a line that can constitute $S(u, v)$. The resulting surface is shown in Figure II:

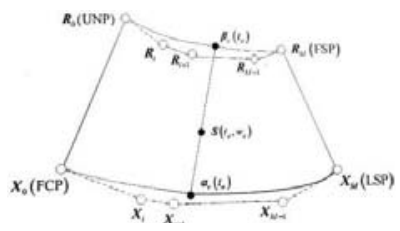

FIGURE II. SURFACE MODELING

When wrapped in a female body, the full 3D clothing prototype shown in Figure III (b) below. Since the resulting $3 \mathrm{D}$ garment prototype is developable, the $3 \mathrm{D}$ garment patch to the 2D patch transformation is achieved by continuous rotation along the corresponding score line.
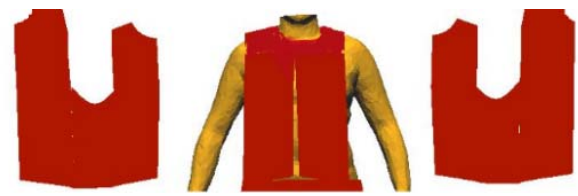

(a)Left side of the flat pattern (b)Designed 3D clothing prototype

(c)Flat pattern or right side

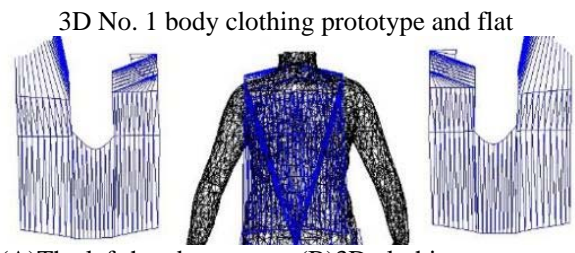

(A)The left-hand geometry (B)3D clothing geometry (C)The right side of the flat geometry

FIGURE III. 3D GARMENT GEOMETRY OF NO. 1 BODY AND ITS FLAT REPRESENTATION

From the simulation results, we can see that the fitting result of the human body and the geometric correspondence between the 3D slice and its flat form can be concluded that, regardless of the changes in body size and fig, the 3D garment design For the scanning of the human body to show good adaptability.

\section{SURface MODELING AND SimUlation}

Fabric simulation based on physical modeling is one of the focuses of computer graphics. Due to the non-linear stressstrain relationship of fabrics, the simulation of fabric is more complicated than ordinary objects. Complex motion of fabrics is reduced to the solution of ordinary differential equations, which opens a new way for better dynamic simulation of flexible fabrics. Euler algorithm is one of the easiest numerical integral methods to solve ordinary differential equations. It is 
simple and has low accuracy. It is not calculated in engineering. The algorithm flow is as follows:

step1. The time domain is divided into discrete time nodes $\mathrm{t} 1, \mathrm{t} 2, \ldots \mathrm{tn}<\mathrm{T}$ by the discrete variable method, and the time step is s, each time node satisfies the formula (10).

$$
t_{i+1}=t_{i}+s,(i=0,1, \ldots, N)
$$

step2.In the Nth discrete points,$t_{i}(i=0,1, \ldots, N)$ to obtain the approximate solution $v_{i}(i=1, \ldots, N)$, Euler algorithm recursive formula:

$$
\begin{aligned}
& v_{i+1}=v_{i}+s * f\left(t_{i}, v_{i}\right) \\
& v_{0}=V_{0}
\end{aligned}
$$

Among them, $v_{0}$ is the initial velocity of the particle, often taking the initial velocity $0, f$ is the acceleration of the particle at time $\mathrm{t}, v_{i}$ is the velocity of the particle at time $t_{i}$, and $\mathrm{s}$ is the time step.

step3.The formula (11) is expanded at time t according to Taylor series,expand as

$$
v(t+s)=v(t)+h^{*} v^{\prime}(t)+\frac{1}{2} v^{\prime \prime}(t)+\cdots \approx v(t)+h^{*} v^{\prime}(t)
$$

As can be seen from equation (12) that the Euler algorithm only takes the first two terms of the Taylor expansion, so the algorithm converges first orderly.

In the simulation experiment, the female in the northern model, which is from 25 to 30 years old, with height of $165 \mathrm{~cm}$ and weight of $55 \mathrm{~kg}$, was selected in the simulation experiment. The simulation experiment was carried out on the threedimensional surface garment prototype constructed in the third chapter. The spring-mass model is added to the garment prototype, and the dynamics is simulated. Dress prototype dress as shown in Figure IV:

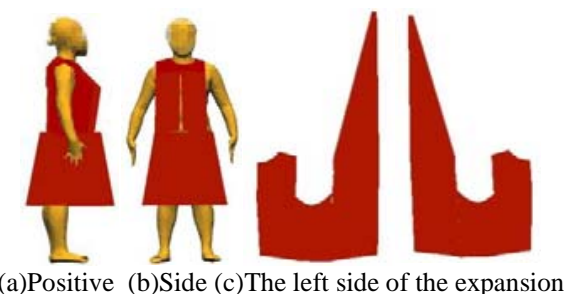

(d) The right side of the expansion

FIGURE IV. MMODELING DRESS EFFECT

From the simulation results: the construction of the threedimensional garment surface of the clothes can be generated to wear a complete model of the body, the right size, the upper body fit with the body better, and lower body due to the body with the threshold of the larger fit Reason, it seems a bit loose. But the simulation results are still relatively good, better expression of the fabric of the realism, to achieve the desired results.

\section{CONCLUSION}

The proposed method for 3D garment prototyping is basically based on a developable Bessel surface. A feature point is introduced on the scanned human body. In addition, not only through the iteration to ensure the construction of Bessel surface control points, and there is no need to solve the complex mathematical equations. 3D clothing prototype is a combination of patches that can be developed, it can be flattened by simple geometric operations for the 2D model, without any distortion. A major drawback with respect to the proposed method is that the continuity of the patch joints is only one level. Because of efficiency and feasibility, can be used to guide the design of clothing.

\section{ACKNOWLEDGMENT}

This work is supported by the Graduate Student Innovation Project of in 2016 (No. 120301990122) and the Education Teaching Reform Project in 2015 (No.ZDJG-1506) of Beijing Institute of Fashion Technology.

\section{REFERENCES}

[1] GerbereTehnology (OL).http//www.gerbertechnology.com.hk/,2003.12

[2] PADSystem(OL).http: //www.padsysetm.com/en/index.html, 2003.12

[3] Karpenko O A,Hughes J F. SmoothSketch: 3D free-formshapes from complex sketches[C] Computer GraphicsProceedings, Aunual Conference Series, ACM SIGGRAPH.New York: ACM Press, 2006: 589-598.

[4] TorayR\&D(OL) .http://www.toray.co.jp/english/products/fash/index.ht $\mathrm{ml}, 2003.12$.

[5] Wang CCL, Chang TKK,Yuen MMF.From laser-scanned data to feature human model:a sye tem based on fuzzy logic concept.ComputAidDes2002;inpress.

[6] C.K. Au, M.F.Yuen: Feature based reverse engineering of mannequin for garment design[J], Computer-Aided Design, 1999 (31): 751-759.

[7] LabsikU,GreinerG.Interpolatory3-subdivision[J].Computer Graphics Forum, 2000, 19(3): 131-138

[8] S.W.Cheng, T. K. Dey, E. Ramos, T. Ray, Sampling and meshing surface with guaranteed topology and geometry, SIAM Journal Computing 37(2007)1199-1227.

[9] SNYDER J M, BARR AH.Ray tracing complex modelscontaining surface tessellations [J]. Computer Graphics, 1987, 21( 4) : 119-128.

[10] CHADWICK J E, HAUMANN D R, PARENT $R$ E. Layeredconstruction for deformable animated characters[C]/ /ACMSiggraph Computer Graphics. ACM, 1989, 23( 3) : 243-252.

[11] LI Yong, HU Min, FU Xiaoli, et al. The research of three-dimensional human body modeling method[J]. Journal ofTextile Research, 2002, 23( 5) : 416-417.

[12] CHEN Lizhen, DENG Zhongmin. Modeling of 3-Dmannequin based on image sequence [J]. KnittingIndustries, 2013( 1) : 54-56. 